Results: Of all the 683 patients, 168 are current smokers, while 108 were ex-smokers and 407 patients never smoked. Mean scores of degree and duration of morning stiffness, overall pain, nocturnal back pain, overall back pain, CRP, BASDAI and BASFI score were all higher in the current smoker group compared to those who had never smoked $(P<0.05)$. In Spearman correlation analysis, pack years of smoking in current smokers were significantly associated with disease duration, morning stiffness (VAS) and duration, nocturnal back pain, overall back pain, CRP, and BASDAI $(P<0.01)$, and overall pain, BASFI and HAQ-S $(P<0.05)$. Conclusions: Cigarette smoking has a dose-dependent relationship with disease activity and correlates with more functional limitation and worse health assessment in patients with AS.

References:

[1] Braun, J. and J. Sieper, Ankylosing spondylitis. Target treatment criteria. Z Rheumatol, 2009. 68(1): p. 30-6.

[2] Ward, M.M., et al., Risk factors for functional limitations in patients with long-standing ankylosing spondylitis. Arthritis Rheum, 2005. 53(5): p. 710-7. Acknowledgements: None.

Disclosure of Interest: None declared

DOI: 10.1136/annrheumdis-2017-eular.2045

\section{FRI0471 PSYCHOLOGICAL DISORDERS, SLEEP DISTURBANCE AND STRESSFUL LIFE EVENTS IN THE PATIENTS WITH ANKYLOSING SPONDYLITIS}

Y. Jiang, M. Yang, Y. Zhang, Z. Liao, Z. Lin, Q. Lv, J. Qi, Q. Wei, S. Cao, J. Gu. Rheumatology, the Third Affiliated Hospital of Sun Yat-sen University, Guangzhou, China

Background: Ankylosing spondylitis (AS) is a chronic inflammatory rheumatic disease that may result in pain, functionally limitation and even less life expectancy. Psychological symptoms not only have a substantial negative impact on the quality of life, but also on the course and outcome of the chronic disorder [1]. AS patients may also suffer from various sleep problems, and pain intensity, anxiety, and depression correlated significantly with poorer sleep quality [2]. Stressful life events have a substantial association with psychological disorders, and there is some evidence indicating that even early life stress constitutes a major risk factor for the subsequent development of mental disorder [3].

Objectives: Our study was to investigate psychological disorders, sleep disturbance and stressful life events in Chinese patients with AS, and to assess the correlation between each two disorders.

Methods: AS patients diagnosed with the modified New York criteria for AS were enrolled from several provinces all over China. Participants were required to complete a set of questionnaires and examinations, including demographic and clinical information, Zung self-rating anxiety scale (SAS), Zung self-rating depression scale (SDS), the Social Readjustment Rating Scale (SRRS) and the Pittsburgh Sleep Quality Index questionnaire (PSQI). The Statistical Package for Social Sciences (SPSS) software version 21 was used for all data management and analysis. The relations between psychological variables, stressful life events, sleep quality and other parameters were examined with Spearman correlation analysis.

Results: Of all the 2772 AS patients, $79.1 \%$ were male patients. $79.5 \%$ were employed. $48.4 \%$ were married. Mean age was $28.99 \pm 8.87$. Mean disease duration was $6.84 \pm 6.78$. Mean SAS score was $49.32 \pm 18.75$. $31.6 \%$ of the patients had anxiety, of which $10.8 \%$ had severe anxiety. Mean SDS score was $54.97 \pm 16.67 .59 .3 \%$ had suffered from depression, of which $11.3 \%$ had severe depression. Mean PSQI score was $6.48 \pm 4.32$. 31.0\% of the patients were poor sleepers. SRRS score was $129.33 \pm 145.06 .35 .3 \%$ had stimulus of psychological and social elements (SPSE), while $14.4 \%$ came across severe SPSE. SAS scores were associated with age, age onset, SDS scores, SRRS scores positively and years of education negatively $(P<0.01)$. SDS scores were associated with age, SAS scores, SRRS scores positively and years of education negatively $(\mathrm{P}<0.01)$. SRRS scores also correlated of less sleep efficiency $(P<0.05)$.

Conclusions: A large number of AS patients were found to have anxiety, depression, sleep disturbance and stressful life events. These problems correlated with each other. Clinicians should pay more attention to psychological disorders and sleep problems in AS patients.

\section{References:}

[1] Gladis MM, Gosch EA, Dishuk NM, Crits-Christoph P (1999) Quality of life: expanding the scope of clinical significance. J Consult Clin Psychol 67: 320-331.

[2] Sayar K, Arikan M, Yontem T (2002) Sleep quality in chronic pain patients. Can J Psychiatry 47: 844-848.

[3] Gourion D (2009) Events of life and links with severe depression at different ages. Encephale 35 Suppl 7: S250-S256.

Acknowledgements: None.

Disclosure of Interest: Y. Jiang: None declared, M. Yang: None declared, Y. Zhang: None declared, Z. Liao: None declared, Z. Lin: None declared, Q. Lv: None declared, J. Qi: None declared, Q. Wei: None declared, S. Cao: None declared, J. Gu Grant/research support from: This project was supported by the National Natural Sciences Foundation of China (31070806), Clinical Subject (2007 and 2010) of Ministry of Public Health of China and from the Foundation of Guangdong province of China (2005A30801005 and 2008B080701086), State Board of Education Fund for the Doctoral (20060558046), Guangzhou Science and Technology Plan Projects (2006Z2-E0221) and 5010 Subject of Sun Yat-sen University (2009-2010)

DOI: 10.1136/annrheumdis-2017-eular.2246

\section{FRI0472 EXTRA-RHEUMATOLOGICAL FEATURES ARE FREQUENTLY ASSOCIATED WITH PERIPHERAL RHEUMATOLOGICAL FEATURES IN AXIAL SPONDYLOARTHRITIS AND INFLUENCE THE CHOICE OF THE ANTI-TNF IN DAILY PRACTICE. AN ANALYSIS OF 519 PATIENTS}

A. Moltó, A. Etcheto, L. Gossec, S. Perrot, N. Boudersa, P. Claudepierre, N. Roux, F. Berenbaum, A. Martin, L. Sparsa, P. Coquerelle, M. Soubrier, M. Dougados. Predict-SpA Study Group, Paris, France

Background: Axial involvement is the predominant feature of axSpA but both extra-articular (EAM)and peripheral manifestations (including synovitis and dactylitis) can be concomitantly observed.

Objectives: To evaluate whether the EAM are associated with peripheral rheumatological features in axSpA and whether these EAM influence the choice of the anti-TNF.

Methods: Study design: Prospective, multicenter, cross-sectional study in France (baseline visit of the Predict-SpA trial). Patients: definite axSpA according to the rheumatologist, requiring an anti-TNF. Data collected: Past or present signs/symptoms of EAM (psoriasis, uveitis and Inflammatory Bowel disease (IBD)), peripheral (arthritis, enthesitis and dactylitis) SpA features, and the anti-TNF prescribed by the treating rheumatologist (according to usual practice). Statistical analysis: the frequency of peripheral involvement and the type of anti-TNF (e.g. monoclonal antibodies vs soluble receptor) were compared in patients with versus (vs) without EAM

Results: Of the 519 patients (females: $46 \%$, age: $42 \pm 12$ years, HLA B27 positive: $65 \%$, X-Ray sacroiliitis: $56 \%$, inflammation on MRI of the sacro lliac Joint: $56 \%$ ), 386 were biologic naïve. A past history or current symptoms of psoriasis, uveitis and IBD was observed in 19\%,17\% and 5\% respectively and a past history or current symptoms of arthritis, enthesitis and dactylitis in $27 \%, 54 \%, 11 \%$, respectively

In patients with vs without psoriasis, the $\%$ of arthritis, enthesitis and dactylitis were $40 \%$ vs $25 \%(p=0.004), 67 \%$ vs $50 \%(p=0.002)$ and $18 \%$ vs $10 \%(p=0.026)$, respectively

In patients with vs without uveitis, the $\%$ of arthritis, enthesitis and dactylitis were $31 \%$ vs $27 \% \quad(p=0.445), 57 \%$ vs $53 \% \quad(p=0.200)$ and $13 \%$ vs $11 \%$ $(\mathrm{p}=0.772)$, respectively

In patients with vs without IBD, the \% of arthritis, enthesitis and dactylitis were $36 \%$ vs $27 \%(p=0.440), 68 \%$ vs $53 \%(p=0.180)$ and $21 \%$ vs $11 \%(p=0.152)$, respectively

Monoclonal antibodies were prescribed as the first biologic in 206 out of the 386 patients $(53 \%)$. This percentage was $56 \%$ vs $58 \%$ and vs $52 \%$ in patients with a current presence of psoriasis vs any history of psoriasis but currently absent and vs without any history of psoriasis, respectively; $p=0.785$. This percentage was $100 \%$ vs $n d \%(n=0)$ and vs $53 \%$ in patients with a current attack of uveitis vs any history of uveitis but currently inactive and vs without any history of uveitis, respectively; $p=0.032$. This percentage was $100 \%$ vs $50 \%(n=2)$ and vs $53 \%$ in patients with a current active IBD vs any history of IBD but currently inactive and vs without any history of IBD, respectively; $p=0.064$.

Conclusions: In this study focused on ax SpA patients, both peripheral rheumatological clinical features and EAM were frequently observed. The peripheral rheumatological manifestations were more frequently observed in case of concomitant psoriasis. Monoclonal antibodies were the preferred anti-TNF in case of a past history (even currently quiescent) of uveitis or IBD in daily practice. Acknowledgements: This study was conducted thanks to an unrestricted grant from MSD

Disclosure of Interest: None declared

DOI: 10.1136/annrheumdis-2017-eular.4713

\section{FRI0473 PATIENT-PHYSICIAN DISCORDANCE IN GLOBAL ASSESSMENT IN 401 EARLY AXIAL SPONDYLOARTHRITIS (AXSPA) PATIENTS FROM THE DESIR COHORT: WHAT IS THE EFFECT ON OUTCOMES AT 3 YEARS?}

C. Desthieux ${ }^{1}$, A. Molto ${ }^{2}$, A. Saraux ${ }^{3}$, B. Fautrel ${ }^{1}$, L. Gossec ${ }^{1} .{ }^{1}$ Rheumatology, Paris 06 University and Pitié-Salpêtrière Hospital; ${ }^{2}$ Rheumatology, Paris Descartes University and Cochin Hospital, Paris; ${ }^{3}$ Cavale Blanche University Hospital, Brest, France

Background: Discordance between patients' and physicians' ratings of disease activity is frequent. We have previously shown this discordance occurs in around $28 \%$ of visits in early axSpA though repeated discordance (over several visits) is less frequent (1).

Objectives: To assess in patients with early axSpA, the impact at 3 years of repeated discordance on treatment prescription, functional incapacity and remission.

Methods: DESIR (Devenir des Spondyloarthropathies Indifférenciées Récentes) is a French, multicenter, longitudinal cohort of patients with early inflammatory back pain suggestive of axSpA (2). Patient and physician global assessment 